\title{
ANÁLISIS DE FALLA DEL DISPOSITIVO DE SUJECIÓN DE UNA FRESADORA
}

\author{
John Jairo Coronado S. *
}

\section{RESUMEN}

En este trabajo se usan las herramientas que nos entrega la ingeniería para determinar las causas que llevaron a la falla del dispositivo de sujeción de una fresadora. Se efectuó el análisis metalográfico del dispositivo y finalmente se dan las recomendaciones necesarias para evitar la falla del dispositivo de sujeción en futuros diseños, especialmente en el proceso de fabricación, el diseño mecánico y el tratamiento térmico del hierro fundido del cual estaba constituida la pieza. 


\section{ABSTRACT}

Different engineering tools were used in this research in order to determine the cause of failure of a milling machine clamp device. The metallographic analysis was done and according to the data the author gave several recommendations to avoid clamp failures in future designs specially in the manufacturing processes, mechanical design and the heat treatment of the grey cast iron of which the clamp was fabricated.

\section{INTRODUCCIÓN}

Cuando se hace un análisis de falla de un elemento mecánico o de un componente estructural, es de suma importancia obtener la mayor información posible de la pieza que falló y conocer el estado de esfuerzos al que estaba sometida en el momento de la falla. Para este análisis se tuvo en cuenta: el material del que estaba construido el dispositivo de sujeción, la naturaleza de los esfuerzos aplicados, la superficie de fractura, el proceso de fabricación y el diseño. El dispositivo de sujeción se muestra en la figura 1.

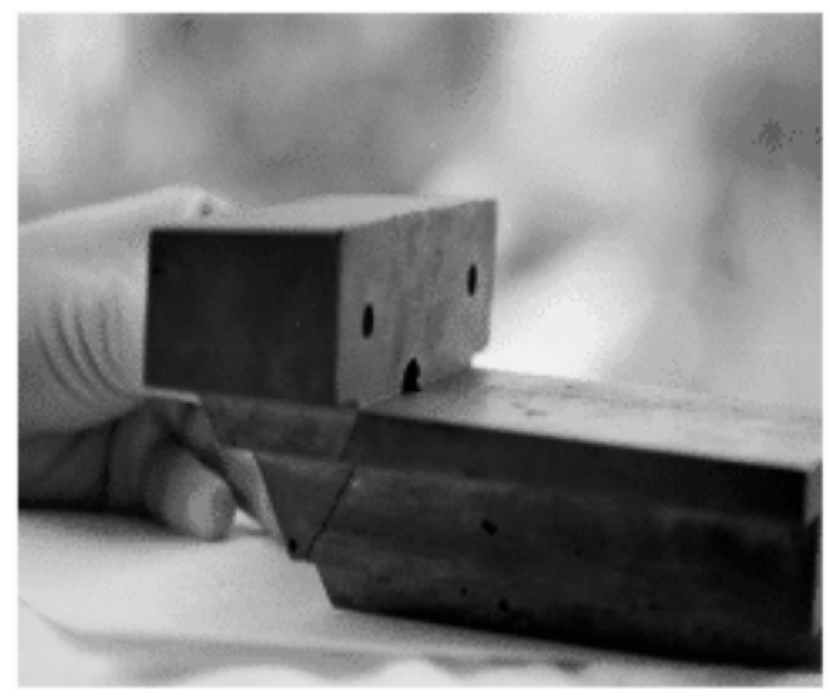

Figura 1. Dispositivo de Sujeción
La figura 2 muestra las vistas lateral y frontal del dispositivo de sujeción con todas sus dimensiones necesarias para los cálculos del centroide, el momento de inercia y el área transversal necesarios para calcular la fuerza máxima que puede aplicarse con seguridad para evitar la falla del componente mecánico.

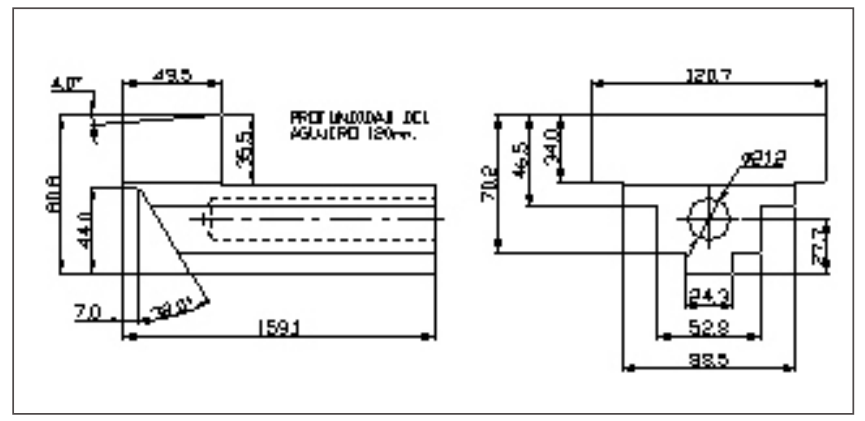

Figura 2. Dimensiones en milímetros del dispositivo de sujeción

La función del dispositivo de sujeción, también conocido como prensa es sujetar piezas en bruto que se van a convertir en un producto final. Para tal fin se usan máquinas herramientas como lo son la fresadora y el taladro. La prensa del presente análisis falló cuando una pieza de acero SAE 1020 se mecanizaba en una fresadora universal, usando una herramienta de corte de acero rápido, conocida como escariador. La Herramienta de corte o fresa en el momento de la falla efectuaba una operación de desbaste, de una cavidad, sobre una pieza previamente torneada.

La prensa es de hierro fundido gris, ASTM A-48, con una resistencia a la tensión de $170 \mathrm{Mpa}$. La prensa presentaba defectos macroscópicos de solidificación como poros. Además presentaba concentradores de esfuerzos debido al diseño que contribuyeron a la fractura final del dispositivo de sujeción.

\section{CÁlCULOS}

El primer paso para evitar otra posible falla es calcular la fuerza máxima que puede aplicarse con seguridad al dispositivo para que no 
sobrepase el esfuerzo normal máximo a tracción de 170 Mpa. La pieza que se va a mecanizar está en contacto con la prensa, la cual produce una carga uniformemente distribuida aplicada a la superficie de la prensa como una reacción a la fuerza ejercida por la pieza sobre la prensa. Para simplificar los cálculos la fuerza distribuida se reemplaza por una fuerza concentrada aplicada en el centroide de la sección transversal de la prensa, cuya magnitud es equivalente a la fuerza máxima que puede aplicarse con seguridad al dispositivo para evitar su falla.

Para la sección transversal mostrada en la figura 3 , se calcula el centroide para ubicar la fuerza resultante. Esta superficie resulta de sumar los tres rectángulos y luego suprimir el circulo. Los datos del área "A", momentos de primer orden " AYY, y las coordenadas del centroide "d," de cada una de las superficies, se presentan en la tabla 1.

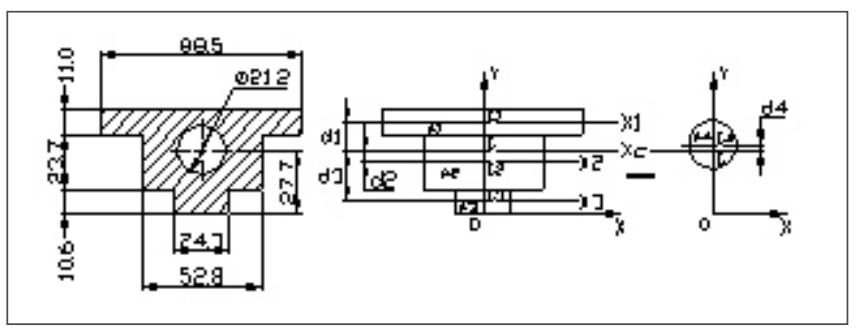

Figura 3. Sección Transversal

\begin{tabular}{|c|c|c|c|c|}
\hline El. & $\begin{array}{c}\text { Área } \\
\left(\mathrm{mm}^{2}\right)\end{array}$ & $\begin{array}{c}Y_{i} \\
(\mathrm{~mm})\end{array}$ & $\begin{array}{c}A_{i} Y_{i} \\
\left(\mathrm{~mm}^{3}\right)\end{array}$ & $\begin{array}{c}d_{i} \\
(\mathrm{~mm})\end{array}$ \\
\hline$A_{1}$ & 973.50 & 39.80 & 38745.30 & 12.3 \\
\hline$A_{2}$ & 1251.36 & 22.45 & 28093.03 & 4.99 \\
\hline$A_{3}$ & 257.58 & 5.30 & 1365.17 & 22.1 \\
\hline$A_{4}$ & -352.99 & 27.70 & -9777.82 & 0.26 \\
\hline$\Sigma$ & 2129.45 & & 58425.68 & \\
\hline
\end{tabular}

Tabla 1. Datos de área y momentos de primer orden (AiYi) de la sección

Dividiendo la resultante del momento de primer orden con respecto al eje coordenado, con la resultante del área de la sección se obtiene el centroide de la sección transversal de la prensa en el eje "Y".

$$
Y=\frac{\Sigma A i Y i}{\Sigma A i}=\frac{58425.68}{2129.45}=27.74 \mathrm{~mm}
$$

\begin{tabular}{|c|c|c|c|}
\hline El. & $\begin{array}{c}\mid x \\
\left(\mathrm{~mm}^{4}\right)\end{array}$ & $\begin{array}{c}\left.A_{i}\right|^{2} \\
\left(\mathrm{~mm}^{2}\right)\end{array}$ & $\begin{array}{c}\mid X_{\mathrm{ci}} \\
\left(\mathrm{mm}^{4}\right)\end{array}$ \\
\hline$A_{1}$ & 9816.13 & 148721.21 & 158537.34 \\
\hline$A_{2}$ & 58573.03 & 31158.99 & 89732.02 \\
\hline$A_{3}$ & 2411.81 & 126260.46 & 128672.27 \\
\hline$A_{4}$ & 9915.47 & 23.86 & 9939.33 \\
\hline
\end{tabular}

Tabla 2. Momentos de inercia de cada sección transversal

El momento de inercia "I $\mid \boldsymbol{}_{c^{\prime \prime}}$ resultante es igual a la suma de los rectángulos menos el circulo por ser un agujero.

$\left|x_{c}=\right| x_{c 1}+\left|x_{c 2}+\right| x_{c 3} \mid x_{c 4}=367002.30 m^{4}$

La distancia del centroide a la fuerza concentrada es $d=17.75+17.86=35.61 \mathrm{~mm}$ y el área total de la superficie es de $2129.45 \mathrm{~mm}^{2}$. De acuerdo a la aplicación de las fuerzas el dispositivo se encuentra sometido a esfuerzos combinados. Si analizamos el eje neutro que pasa por el centroide de la sección transversal, encontramos que la parte superior se encuentra sometida a tracción debido al momento flector " $M$ ", mientras que la parte inferior se encuentra a compresión. La sección crítica se encontró en la parte superior donde se suman los esfuerzos debido a la carga axial "F" $y$ al momento flector.

$$
\begin{gathered}
\sigma=\sigma_{\text {flexión }}+\sigma_{\text {tracción }} \\
\sigma=\frac{M y}{I x_{c}}+\frac{F}{A}=\frac{(F d) y}{I x_{c}}+\frac{F}{A}
\end{gathered}
$$

Donde " $y$ " es la distancia del eje neutro a la fibra exterior, su valor es de $17.86 \mathrm{~mm}$. Despejando la fuerza tenemos:

$$
\mathrm{F}=\frac{d y}{I x_{c}}+\frac{1}{A}=77183.29 \text { Newton }
$$


La fuerza máxima que puede aplicarse con seguridad al dispositivo es de 77.18 KM.

\section{TIPO DE FRACTURA}

La superficie de fractura no presenta deformación plástica y su apariencia es de color grisáceo, típica del hierro fundido gris del cual está constituida la prensa. La fractura empezó en la parte superior donde hay un ángulo recto entre dos superficies que aumentó la concentración de esfuerzos. Además la prensa presentó un poro de gran tamaño en el ángulo recto, por donde se propagó la fisura. Además la excesiva profundidad del agujero que contribuyó a concentrar los esfuerzos y a disminuir el área efectiva que se opone a las fuerzas externas.

\section{MATERIAL}

El material usado en la prensa fue de hierro fundido gris debido a su bajo costo, facilidad para ser fundido en grandes cantidades y su excelente maquinabilidad. Pero las desventajas que presenta el hierro fundido gris son su fragilidad y baja resistencia a la tracción. A ésta prensa no se le practicó ningún tratamiento térmico, es conveniente practicar un tratamiento térmico para acabar las tensiones internas, ya que el enfriamiento avanza con diferentes velocidades por toda la sección de la pieza fundida. Las tensiones residuales resultantes pueden reducir la resistencia mecánica disponible de la pieza de fundición, producir distorsiones después del enfriamiento y puede contribuir a la formación de grietas.

Las condiciones de servicio, permiten utilizar este mismo material corrigiendo los factores $y$ variables del proceso de fundición con el cual se obtuvo inicialmente. También es factible utilizar otro material con mejores propiedades mecánicas como el acero, pero teniendo en cuenta el proceso de fabricación y el costo.

\section{PROCESO DE FABRICACIÓN}

El proceso de fabricación usado para producir la prensa fue el de fundición en molde de arena y posteriormente se obtuvieron las dimensiones finales por desprendimiento de material. Se pueden presentar diferentes zonas de temperatura en la pieza con respecto al bebedero y al alimentador, lo cual afectó la calidad del material.

Debido a la gran cantidad de poros observados es evidente que no se tuvo un buen control en la calidad de la fundición. En el proceso de fundición se deben tener en cuenta: velocidad de enfriamiento, tiempo de colada, preparación adecuada de los moldes para evitar que los granos de la arena queden adheridos a la pieza.

\section{DISEÑO MECÁNICO}

Puesto que el ángulo recto contribuye a la concentración de esfuerzos se recomienda hacer un radio de $5 \mathrm{~mm}$. Para no interferir en la sujeción de la pieza a mecanizar se debe usar otra superficie de apoyo unida con tornillos a la prensa. El agujero ciego se puede disminuir de longitud de forma tal que debajo del filete exista material ya que de lo contrario este agujero concentra los esfuerzos en la zona critica.

\section{EXPERIMENTACIÓN}

Se analizaron dos muestras del material de la prensa uno cerca del área de falla y el otro alejado. Las muestras se observaron en el microscopio óptico a diferentes aumentos. La figura 4 presenta la microestructura a $100 x$ de la prensa en una zona alejada de la falla, se puede apreciar la distribución de las hojuelas de grafito de tamaño pequeño al azar y segregación ínter dendrítica, tipo E, debido aun rápido enfriamiento. 


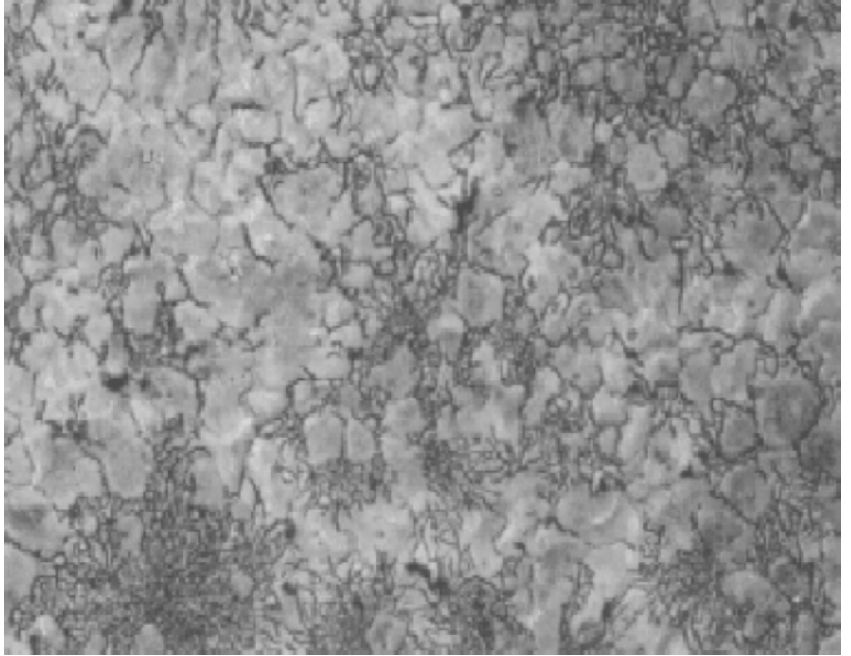

Figura 4. Microestructura a $100 \times$ alejada de la zona de falla

La figura 5 presenta la microestructura a $500 x$ alejada de la falla, muestra una matriz perlítica fina con trazos de ferrita. El grafito aparece como muchas placas irregulares, alargadas y curvas, las cuales le dan al hierro fundido gris su característica fractura de color grisáceo o negrusco.

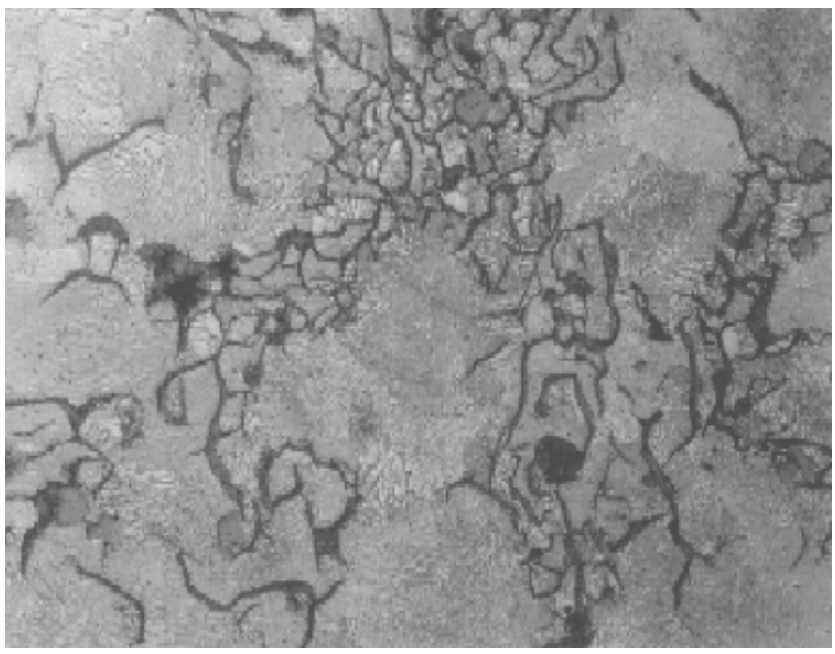

Figura 5. Microestructura a $500 \times$ alejada de la zona de falla

La figura 6 presenta la microestructura a $100 x$ cerca de la falla. Las hojuelas de grafito se encuentran distribuidas sobre la matriz perlítica rompiendo la continuidad de ésta. Los esfuerzos se concentran conduciendo a una disminución de la resistencia y de la ductilidad. También se pueden apreciar micro rechupes debido a un deficiente control de calidad en el proceso de fundición.

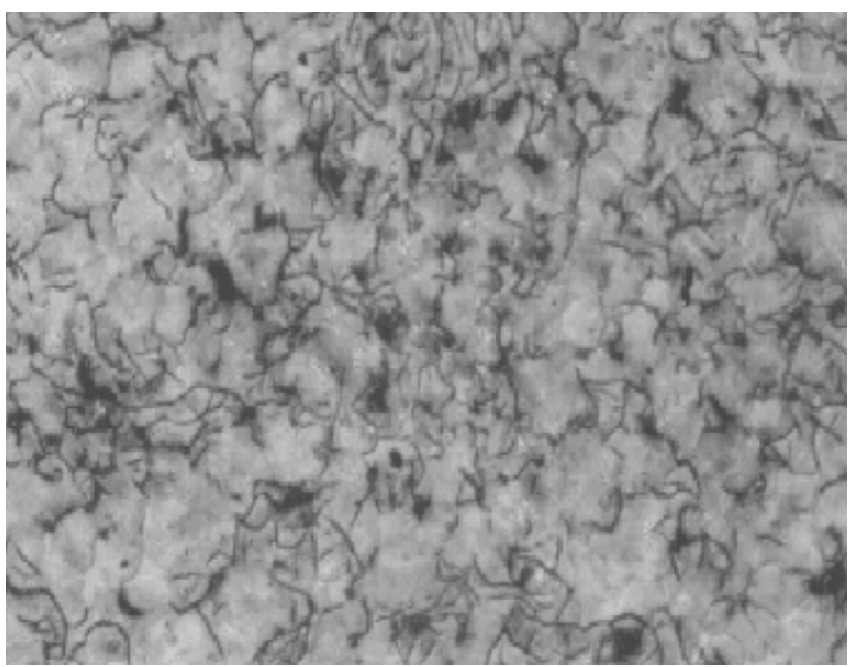

Figura 6. Microestructura a $100 \times$ cerca de la zona de falla

La figura 7 presenta la microestructura a 500x del área de falla, muestra una matriz de perlita gruesa, lo cual indica que en esta zona el enfriamiento fue más lento. La microestructura también presenta un aumento en la cantidad de ferrita conduciendo a una disminución de la resistencia en dicha zona. El lento enfriamiento produjo hojuelas de grafito más grandes en la zona de falla, conduciendo a una mayor concentración de esfuerzos, ya que interrumpen la continuidad de la matriz perlítica, disminuyendo la resistencia y la ductilidad.

Además se encontraron inclusiones no metálicas ampliamente dispersadas de sulfuro de manganeso, que no afectan las propiedades de la fundición. También se encontró gran cantidad de esteadita. Cuando hay excesiva cantidad de fósforo en la fundición se combina con el hierro para formar fosfuro de hierro, el cual constituye un eutéctico ternario con la cementita y la austenita a temperatura ambiente. El eutéctico primario se 
conoce esteadita y es relativamente frágil, rodeando las dendritas primarias de austenita.

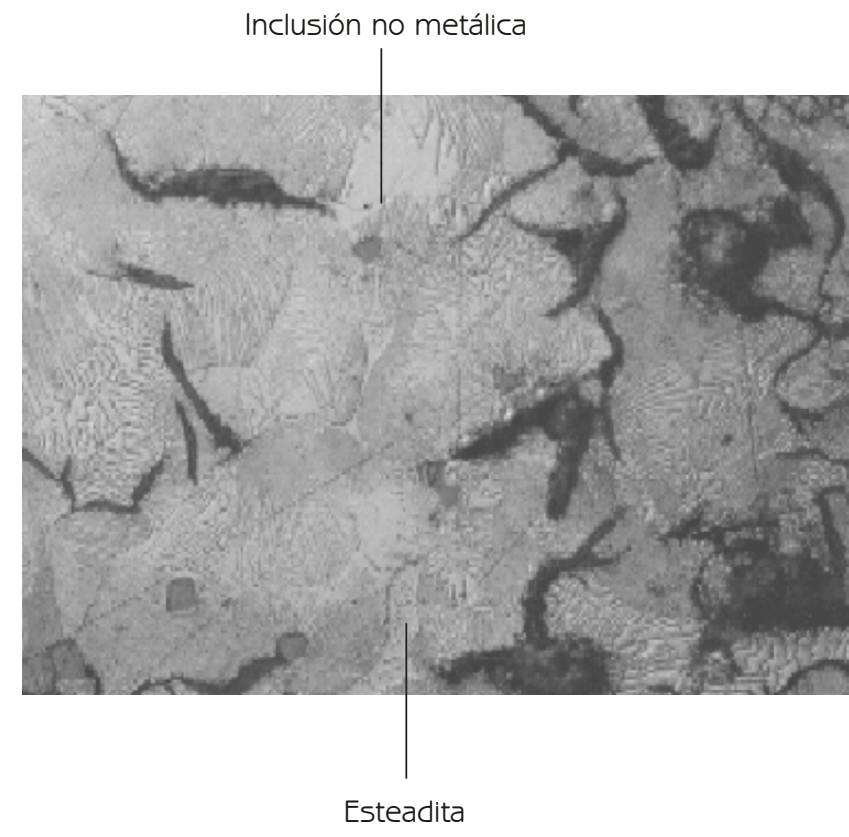

Figura 7. Microestructura a $500 \times$ cerca de la zona de falla

\section{CONCLUSIONES}

El proceso de fundición empleado no contó con el control adecuado, debido a las imperfecciones de la prensa como por ejemplo los poros presentados y la formación de esteadita.

La prensa presenta deficiencias en el diseño, como ángulos rectos en los cambios de sección y excesiva longitud del agujero que concentraron los esfuerzos en la prensa en el área de falla.

\section{RECOMENDACIONES}

La fuerza máxima que puede aplicarse con seguridad al dispositivo para evitar su falla es de $77.18 \mathrm{KM}$.

Controlar el porcentaje de fósforo en el hierro para obtener unas buenas propiedades, ya que el alto contenido de fósforo reduce la tenacidad y lo hace frágil.
Cambiar el diseño del dispositivo usando radio en la unión de las dos secciones de la prensa y disminuir la longitud del agujero, lo cual no afecta la funcionalidad de la prensa.

Es recomendable aplicar un tratamiento térmico para aliviar las tensiones internas, conocido como recocido, y consiste en calentar la pieza a una temperatura de $550^{\circ} \mathrm{C}$ y enfriar lentamente.

Se debe hacer un cuidadoso control en la rapidez de enfriamiento y el contenido de silicio, para garantizar un hierro perlítico gris de alta resistencia. Esto se puede lograr grafitizando la cementita eutéctica y proeutectoide.

Se puede mejorar la calidad del hierro fundido gris diminuyendo el tamaño de las hojuelas y con una mejor distribución. Esto de puede lograr con la adición de inoculante.

Realizar una buena desgasificación para evitar que se presente segregación, porosidad y cavidades centrales por contracción durante la solidificación.

\section{BIBLIOGRAFÍA}

BROOKS, Charly R, "Metallurgical Failure Analysis". Ed. Mc Graw Hill.

METALS HAMDBOK, "Metallographic and Microestructures". Minth Edition. Volume 9.

VALEMCIA, Asdrúbal, "Tecnología del Tratamiento Térmico de los Metales". Ed. Universidad de Antioquia.

AVMER, Sydney H., "Introducción a la Metalurgia Física". Editorial Mc Graw Hill.

THORMTOM, Peter A., "Ciencia de Materiales para Ingeniería". Ed. Prentice Hall Hispanoamericana, S.A

GROOVER, Mickell. , "Fundamentos de Manufactura Moderna". Ed. Prentice Hall.

BEER, Ferdinand P., "Mecánica Vectorial para Ingenieros". Ed. Mc Graw Hill.

PYTEL, Andrew. , "Resistencia de Materiales". Editorial Harla.

SHIGLEY, Mc Joseph, "Diseño en Ingeniería Mecánica". Ed. Graw Hill. 